\title{
Monitoring priority pollutants in a sewage treatment process by dichloromethane extraction and triolein-semipermeable membrane device (SPMD)
}

\author{
Yi Wang *, Zijian Wang, Mei Ma, Chunxia Wang, Zheng Mo \\ State Key Laboratory of Environmental Aquatic Chemistry, Research Center for Eco-Environmental Sciences, \\ Chinese Academy of Sciences, Beijing 100085, People's Republic of China
}

Received 13 September 1999; accepted 27 March 2000

\begin{abstract}
Semipermeable membrane devices containing a neutral lipid triolein (triolein-SPMD) and conventional dichloromethane extraction were used to monitor the presence and concentrations of priority organic pollutants in a sewage treatment process in Beijing, China. Both samples gave similar information on the presence of target PCB congeners, PAHs, Organochlorine pesticides and substituted benzenes in sewage at all sites. After 20 days' sampling, the concentrations of contaminants in triolein of SPMD were much higher than those in dichloromethane extracts, which resulted in easier analysis, improved the detection limits, and increased the accuracy. Previous field mean sampling rates for SPMD were used to estimate concentrations of PCB congeners in sewage, which compared to their determined concentrations by dichloromethane extraction. The consistency and superiority of SPMD technology were proved for the detection of priority organic contaminants from sewage. Lower removal rates for PCB congeners and PAHs coincided with the persistence of them in environment. More attention should be paid to urban sewage treatment process adopting activated-sludge method, where priority organic pollutants might not be removed, even present higher concentrations after treatment. (C) 2001 Elsevier Science Ltd. All rights reserved.
\end{abstract}

Keywords: SPMDs; Sewage treatment process; PCB; PAH; Monitoring

\section{Introduction}

Triolein-Semipermeable membrane device (SPMD) consists of a thin film of a neutral lipid triolein, enclosed in thin-walled layflat tubing made of low-density polyethylene or other nonporous polymers. The characteristic free volume of polymeric films allows dissolution of organic molecules having a cross-sectional diameter of less than $1 \mathrm{~nm}$ into the membrane and diffusion through

\footnotetext{
${ }^{*}$ Corresponding author. Present Address: Department of Geological Sciences, Brown University, Providence, RI 029121846, USA.

E-mail address: ywang@mitkem.com (Y. Wang).
}

the nonporous membrane (Huckins et al., 1993). SPMDs are passive samplers that provide a means of determining the dissolved portion of lipophilic organic compounds in the aquatic environment. This sampling process is similar to the diffusion of lipophilic organic compounds through biological membranes, such as contaminant transfer through respiratory lamellae of fish (Huckins et al., 1996a).

SPMDs have been used by researchers to monitor trace hydrophobic organic pollutants in many different aquatic environments. Results from these studies demonstrated that SPMDs were effective in concentrated contaminants, such as PCBs, PAHs, etc. (Bennett et al., 1996; Huckins et al., 1996b; Hofelt and Shea, 1997; Huckins et al., 1997; Sabaliunas and Sodergren, 1997; 
Wang et al. 1999). Yet much more background data are still needed to determine if SPMDs are applicable in diverse environmental monitoring programs.

In this work, triolein-SPMDs were deployed along a sewage treatment process in Beijing, China, for assessing the contamination of priority organic pollution in sewage, at the same time, for testing SPMD's applicability in sewage. Water samples were also extracted by using conventional dichloromethane for effect comparison.

\section{Experimental}

\subsection{Sampling sites}

The studied sewage treatment process in Beijing adopts an international popular secondary treatment process. The treatment effect seems good since BOD, COD and SS in treated sewage decrease from $80 \%$ to $95 \%$. However, presence and concentrations of trace priority organic pollutants in sewage have not been studied yet, which should be of great importance considering their potential toxicity and environmental risk.

In this study, four sampling sites along the process were selected, as shown in Fig. 1. In brief, site 1 receives the untreated sewage from Beijing city (population: more than 10,000,000), site 2 receives sewage treated by aeration and sedimentation, site 3: sewage after activated-sludge treatment, site 4: treated sewage for discharge after the final sedimentation. There were no major differences in current velocity among the above four sites (below $0.5 \mathrm{~m} / \mathrm{s}$ ) and were relatively constant during the sampling period. Sewage temperature ranged from $12^{\circ} \mathrm{C}$ to $13.5^{\circ} \mathrm{C}$.
The sampling distance is approximately $1 \mathrm{~km}$ and the time for sewage flowing through was estimated to be less than $1 \mathrm{~h}$. It could be inferred that SPMDs at all sites sampled the same sewage and could record most of the discharging events during the 20-day exposure.

\subsection{Assembly of SPMDS}

Triolein obtained from Sigma Chemical, St. Louis, MO, USA, was $99 \%$ pure. Layflat LDPE, purchased from Beijing Plastics, was a 2.5 -cm wide, untreated (pure PE, no slip additives, etc.) clear tubing. Its wall thickness ranged from 50 to $55 \mu \mathrm{m}$. The PE tubing was cut into pieces of $20 \mathrm{~cm}$ in length, soaked in cyclohexane for $24 \mathrm{~h}$ to remove trace contaminants, and dried with ultra-pure nitrogen. The total mass of tubing was $1.05 \mathrm{~g}$, and the ratio of mass of tubing to volume of solvent was $0.01 \mathrm{~g} /$ $\mathrm{ml}$. Then $40 \mu \mathrm{l}(36.4 \mathrm{mg})$ of triolein was coated onto the inner part of the tubing before the ends were sealed with a commercial sealer (Wang et al., 1999). The ratio of SPMD surface area to triolein volume is $2500 \mathrm{~cm}^{2} / \mathrm{ml}$, which was five times higher than that of standard SPMD configuration (Huckins et al., 1996a). Every SPMD was fixed in a fresh solvent-cleaned PVC plastic-meshed cage $\left(5 \times 3 \times 25 \mathrm{~cm}^{3}\right)$.

\subsection{SPMD deployment}

After preparation, SPMDs were placed into a low gas permeable and portable cooler, which was solventcleaned and cooled with dry ice. During October 1997, Four SPMDs were deployed at each site along the sewage treatment process. SPMDs were suspended $1 \mathrm{~m}$ below the surface. After a 20-day exposure, the SPMDs

1 2 $3 \quad 4$
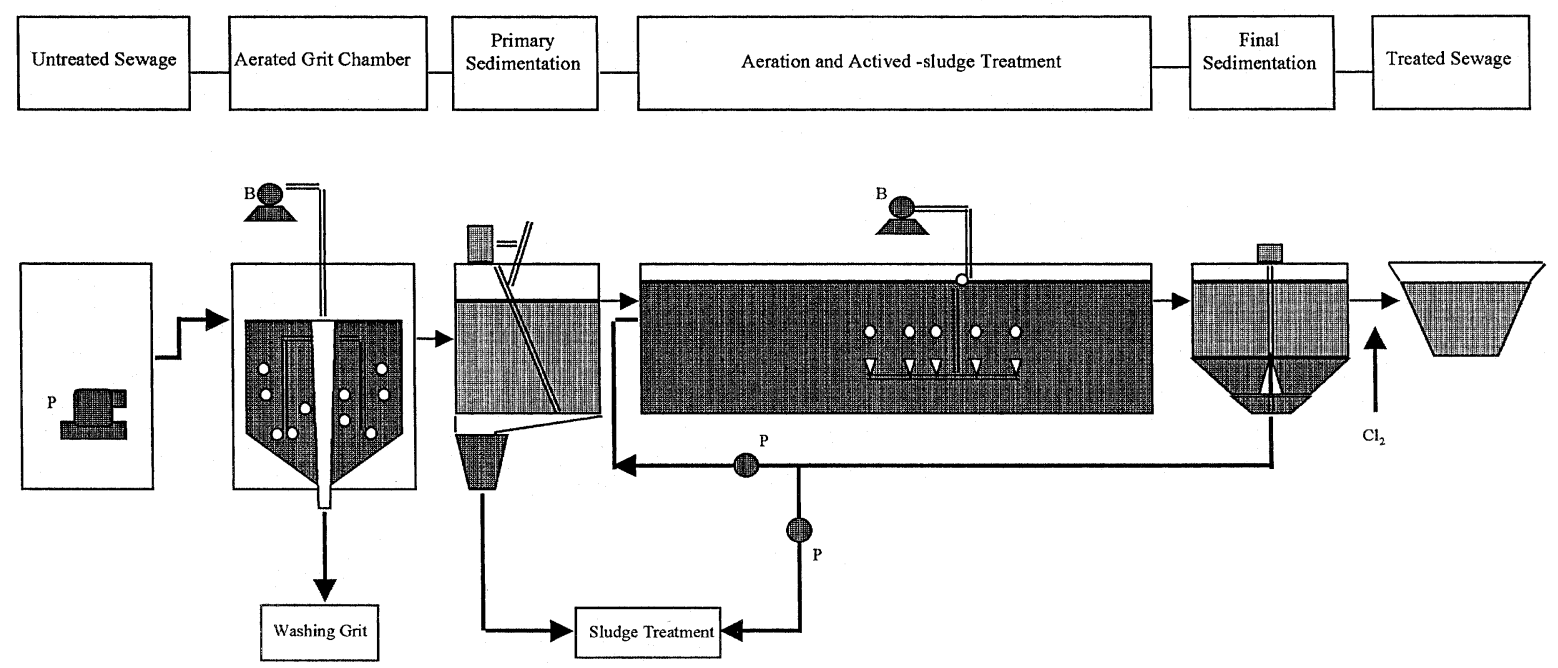

Fig. 1. Location of sampling sites along the sewage treatment process in Beijing, China. 
were retrieved, placed into the portable cooler on dry ice again, and immediately transported to the laboratory for sample processing and residue enrichment. Three controls of SPMDs were used during deployment, retrieve and transportation.

Due to the hardness of water sampling, 2.51 of sewage samples at each site was collected only once by the end of SPMD exposure, which should have been carried out continuously in order to make a good comparison.

\subsection{Sample processing and residue enrichment}

Each SPMD was rinsed with tap water, distilled water, $1 \mathrm{~N} \mathrm{HCl}$ solution for $30 \mathrm{~s}$ to remove surfacebiofouled residues (Wang et al., 1999). Then it was rinsed with distilled water, dried with clean paper, and transferred into a beaker containing $100 \mathrm{ml}$ cyclohexane. It was dialyzed at $13^{\circ} \mathrm{C}$ for $48 \mathrm{~h}$ in the darkness. The solvent was reduced in volume to about $1 \mathrm{ml}$ using a gentle stream of high-purity nitrogen. Then it was cleaned up by passing anhydrous sodium sulfate (filled in a column of $4 \mathrm{~cm}$ long), silica gel (60-80 mess, deactivated, $4 \mathrm{~cm}$ long) columns. Extra volume of pure cyclohexane was used to elute target compounds remaining on the column. Finally, the extract was treated with copper wool to remove sulfur, filtered through glass filters, reduced in volume (to about $0.5 \mathrm{ml}$ ) again, and diluted to $1 \mathrm{ml}$.

Sewage sample was filtered through $0.45 \mu \mathrm{m}$ pretreated microporous membranes (Beijing Reagent) to remove undissolved matters. Each $500 \mathrm{ml}$ of water was extracted with $40 \mathrm{ml}$ of solvent in three steps and each of $20,10,10 \mathrm{ml}$, respectively. A total of 21 of the filtered sewage was extracted with $160 \mathrm{ml}$ of dichloromethane. It is known that compounds of interest (PCB, PAH) may sorb to filter membranes more or less. Hence before filtration, those membranes were pretreated in DI water and water-bathed $\left(60^{\circ} \mathrm{C}\right)$ for $8 \mathrm{~h}$. After filtration, they were collected for each site, rinsed with enough DI water, then Soxhlet extracted by dichloromethane for $12 \mathrm{~h}$ to check the degree of absorption. All extract was cleaned up in the same manner as that of SPMD described earlier, and blown down in volume using highpurity nitrogen, then quantitatively diluted to $1 \mathrm{ml}$.

\subsection{Chemical analysis}

Target chemicals included 57 PCB congeners (from di-chlorinated biphenyl to hepta-chlorinated biphenyl), three PAHs (naphthalene, phenanthrene and anthracene), four organochlorine pesticides (allethrin, chlordane, trans-chlordane and endosulfan), two herbicides (atrazine and acetochlor), and 15 substituted benzenes (toluene, xylene, nitrobenzene, chlorophenol, etc.).

Sample analysis was performed on a gas chromatography-mass spectrometry (GC-MS, HP 5890 II/ 5971A, HP-5 column, $30 \mathrm{~m} \times 0.25 \mathrm{~mm}$ id $\times 0.25 \mu \mathrm{m}$, connected with another HP-5 column, $25 \mathrm{~m} \times 0.25 \mathrm{~mm}$ id $\times 0.33 \mu \mathrm{m}$, carrier gas: He). External standards like Clophen A50 were used in quantitative analysis for PCB congeners and other target pollutants. To avoid interference of polyethylene waxes and lipids, selected ion monitoring mode (SIM) was used (Frame, 1997).

\subsection{Quality control}

Three field controls of SPMDs accompanied the SPMD sampler arrays during their deployment, retrieve and transportation. They were processed and analyzed exactly as the deployed samples. No PCBs and other pollutants were present in SPMD procedural controls. One SPMD blank (a freshly prepared SPMD) and two reagent blanks $(100 \mathrm{ml}$ of cyclohexane and $100 \mathrm{ml}$ of dichloromethane, blow down to $1 \mathrm{ml}$, respectively) were also taken through the entire clean up and enrichment procedure. No chemicals of concern were found in these blanks either. By standard addition, (five blank waters, 2 1 each; five blank triolein-SPMDs, same size as field ones) the recoveries of PCB congeners (level: $1 \mu \mathrm{g} / \mathrm{ml}$ ) and other pollutants (level: $20 \mu \mathrm{g} / \mathrm{ml}$ ) were between $60 \%$ and $93 \%$, with the relative standard deviation less than $20 \%$.

\section{Results and discussion}

\subsection{Variation of routine targets along the sewage treatment process}

Table 1 gave the results of routine targets along the sewage treatment process determined during the SPMD

Table 1

Variation of routine targets along four sites determined during SPMD exposure

\begin{tabular}{llllll}
\hline Sites & Colority & SS (mg/l) & pH & $\mathrm{NH}_{3}-\mathrm{N}(\mathrm{mg} / \mathrm{l})$ & COD (mg/l) \\
\hline 1 & 222 & 50 & 69 & 17.6 & 101.2 \\
2 & 168 & 28 & 6.9 & 17.9 & 85.8 \\
3 & 39 & 8 & 6.9 & 1.4 & 23.4 \\
4 & 9 & 84 & 6.9 & 0.1 & 14.7 \\
Removal rate $(\%)$ & 95.9 & & 99.4 & 85.5 \\
\hline
\end{tabular}


exposure. It is obvious that all targets declined after treatment except $\mathrm{pH}$ value. For example, the removal rates of SS and COD were over $84 \%$, for colority and $\mathrm{NH}_{3}-\mathrm{N}$, higher than $95.9 \%$. These targets meant treated sewage came up to National Standards of Surface Water (GB3838-88, P.R. China).

\subsection{Monitoring effect comparison by using dichloro- methane extraction and triolein-SPMD}

Tables 2 and 3 gave the presence and concentrations of target pollutants in SPMDs and extracts of filtered sewage. In Table 2, three PAHs could be detected in all samples. For PCB congeners, 23 congeners could be found in SPMD, while in dichloromethane extracts, eight of these 23 congeners were not detected because of their very low concentrations in sewage (PCB 61, 83, 84, $86,87,99,101,176)$ and volume limitation of liquidliquid extraction. Analysis for PCB and PAH in soxhlet extracts of filter membranes showed very low neglectful levels of target compounds on membranes (less than $5 \%$ ). So no further calculation was taken. In Table 3, eight substituted benzenes including toluene, xylene, 2,4dinitrotoluene were detected in SPMDs and sewage extracts, while four OCPs and other target contaminants were not found in both samples.

In sewage extracts, the concentrations of PCB congeners were between 0.1 and $32 \mathrm{pg} / \mathrm{ml}$, while after only

Table 2

Monitoring effect comparison for PAHs and PCB congeners in sewage by using dichloromethane extraction and Triolein-SPMD technology

\begin{tabular}{|c|c|c|c|c|c|c|c|c|}
\hline \multirow[t]{2}{*}{ Contaminants } & \multicolumn{2}{|l|}{ Site 1} & \multicolumn{2}{|c|}{ Site 2} & \multicolumn{2}{|c|}{ Site 3} & \multicolumn{2}{|c|}{ Site 4} \\
\hline & $\overline{W^{\mathrm{a}}}$ & $S^{\mathrm{b}}$ & $\bar{W}$ & $S$ & $\bar{W}$ & $S$ & $W$ & $S$ \\
\hline \multicolumn{9}{|l|}{$P A H s$} \\
\hline Naphthalene & 0.2 & 34 & 1.4 & 29 & 1 & 44 & 1 & 72 \\
\hline Phenanthrene & 0.2 & 10 & 0.2 & 26 & 0.4 & 7 & 0.2 & 12 \\
\hline Anthracene & 0.2 & 20 & 0.2 & 7 & 0.6 & 11 & 0.6 & 16 \\
\hline \multicolumn{9}{|l|}{ PCB congeners } \\
\hline $4,5,15,18,19,21$ & $-^{\mathrm{c}}$ & - & - & - & - & - & - & - \\
\hline $28,31,37,40,41,44$ & - & - & - & - & - & - & - & - \\
\hline 25 & - & 46 & 0.1 & 78 & 1 & 193 & - & 439 \\
\hline $46,47,49,70,82$ & - & - & - & - & - & - & - & - \\
\hline 45 & - & 1 & - & 36 & - & 13 & - & 138 \\
\hline 52 & 3.9 & - & 2.4 & 54 & - & 50 & 2 & 21 \\
\hline 61 & - & - & - & 4 & - & - & - & 5 \\
\hline 83 & - & 46 & - & 3 & - & 3 & - & 2 \\
\hline 84 & - & 4 & - & 4 & - & 5 & - & 78 \\
\hline 86 & - & 2 & 0.4 & 2 & 1.2 & 3 & - & 2 \\
\hline 87 & - & 2.5 & - & 8 & 4.4 & 3 & - & 2 \\
\hline 95 & 1 & 31 & - & 13 & 2.3 & 387 & 2 & 35 \\
\hline 99 & - & 0.6 & - & 1 & 0.4 & 1 & - & 1 \\
\hline 101 & - & 1.9 & - & 13 & 2 & 3 & - & 3 \\
\hline $105,129,134,136$ & - & - & - & - & - & - & - & - \\
\hline 110 & - & 0.6 & 3 & 4 & 2 & 1 & 9 & 1 \\
\hline 118 & 6.6 & 81 & 6.6 & 136 & 18 & 298 & 13 & 111 \\
\hline 120 & - & 0.6 & 1.7 & 12 & - & 2 & 5 & 1 \\
\hline 128 & 0.5 & 6.3 & 1 & 12 & 1 & 8 & 1 & 5 \\
\hline 132 & 14 & 93 & 15.2 & 26 & 15 & 248 & 19 & 152 \\
\hline 135 & 2.4 & 29 & 2.4 & 49 & 7 & 108 & 5 & 40 \\
\hline $138,146,149,156$ & - & - & - & - & - & - & - & - \\
\hline $174,177,178,179$ & - & - & - & - & - & - & - & - \\
\hline 151 & 12 & 40 & 6.8 & 15 & 9 & 155 & 9 & 32 \\
\hline 153 & 7 & 43 & 7 & 12 & 7 & 116 & 9 & 71 \\
\hline 170 & 17 & - & 17 & - & 17 & 298 & 18 & 179 \\
\hline 176 & - & 6.9 & - & 3 & - & 4 & - & 3 \\
\hline 180 & 10 & 26 & 20 & 12 & 10 & 169 & 32 & 102 \\
\hline
\end{tabular}

${ }^{\mathrm{a}}$ Sewage, extracted with dichloromethane, 2 1, unit: pg/ml (PAHs), pg/ml (PCB congeners), expressed as concentration in dichloromethane extract.

${ }^{\mathrm{b}}$ SPMD, 20-day exposure, unit: pg/ml (PAHs), pg/ml (PCB congeners), expressed as concentration in triolein of SPMD.

${ }^{\mathrm{c}}$ Undetectable. 
Table 3

Monitoring effect comparison for substituted benzenes and other target contaminants in sewage by using dichloromethane extraction and Triolein-SPMD technology

\begin{tabular}{|c|c|c|c|c|c|c|c|c|}
\hline \multirow[t]{2}{*}{ Contaminants } & \multicolumn{2}{|c|}{$\underline{\text { Site } 1}$} & \multicolumn{2}{|l|}{$\underline{\text { Site } 2}$} & \multicolumn{2}{|l|}{$\underline{\text { Site } 3}$} & \multicolumn{2}{|c|}{ Site 4} \\
\hline & $W^{\mathrm{a}}$ & $S^{\mathrm{b}}$ & $W$ & $S$ & $W$ & $S$ & $W$ & $S$ \\
\hline Toluene & 0.2 & 36.3 & 136 & 25 & 100 & 73.8 & 9.2 & 119 \\
\hline Ethylbenzene & $-^{c}$ & - & - & - & - & - & - & - \\
\hline$p$-Xylene & 0.2 & 2.5 & 7.7 & 1.3 & 14 & 1.7 & 0.2 & 25 \\
\hline$o$-Xylene & 0.2 & 3.8 & 19 & 2.5 & 22 & 3.3 & 1.6 & 31 \\
\hline Phenol & - & 0.8 & 44 & 3.3 & 2.4 & 1.1 & 2.4 & 8.7 \\
\hline Aniline & 1.4 & - & 4.6 & - & 0.2 & 4.8 & 1.4 & 2.9 \\
\hline 2-Chlorophenol & 0.2 & - & - & - & - & - & - & - \\
\hline Nitrobenzene & 0.2 & - & - & - & - & - & - & - \\
\hline 2,4-Dichlorophenol & - & - & - & - & - & - & - & - \\
\hline 2,3-Dichlorophenol & - & - & - & - & - & - & - & - \\
\hline 2,4,6-Trichlorophenol & - & - & - & - & 0.2 & - & - & - \\
\hline 2,6-Dinitrotoluene & 0.2 & - & 0.2 & 340 & 3.2 & 6.7 & - & 1.6 \\
\hline 4-Nitrophenol & 0.4 & 1.9 & 0.6 & 15.6 & 1.8 & 3.3 & 1.8 & 2.5 \\
\hline 2,4-Dinitrotoluene & 0.4 & 105 & 1 & 297.5 & 0.4 & 30 & 0.6 & 54 \\
\hline Hexachlorobenzene & 0.8 & - & - & - & - & - & - & - \\
\hline Atrazine & - & - & - & - & - & - & - & - \\
\hline Acetochlor & - & - & - & - & - & - & - & - \\
\hline Decosane & - & - & - & - & - & - & - & - \\
\hline Allethrin & - & - & - & - & - & - & - & - \\
\hline Chlordane & - & - & - & - & - & - & - & - \\
\hline Trans-chlordane & - & - & - & - & - & - & - & - \\
\hline Endosulfan & - & - & - & - & - & - & - & - \\
\hline
\end{tabular}

${ }^{a}$ Sewage, extracted with dichloromethane, volume: 21 , unit: $\mu \mathrm{g} / \mathrm{ml}$, expressed as concentration in dichloromethane extract.

${ }^{\mathrm{b}}$ SPMD, 20-day exposure, unit: $\mu \mathrm{g} / \mathrm{ml}$, expressed as concentration in triolein of SPMD.

${ }^{\mathrm{c}}$ Undetectable.

20 days' sampling, their concentrations in triolein of SPMDs came up to as high as $439 \mathrm{pg} / \mathrm{ml}$. For PAHs and other detected substituted benzenes, their concentrations in SPMD were 10-100 times higher than that in dichloromethane extracts. Much higher concentrations of target chemicals in SPMD resulted in easier analysis, improved the detection limits, and increased the accuracy.

Above results inferred the consistency and superiority of SPMD technology compared to conventional dichloromethane extraction technology for the detection of priority organic contaminants from sewage.

\subsection{Variation of target priority organic pollutants along the sewage treatment process}

Fig. 2 showed that removal rates for PCB congeners and PAHs are less than $10 \%$, while for most substituted benzenes, they are less than $20 \%$, some even presented higher concentrations after treatment (shown in Fig. 3). On the one hand, above results coincided with the persistence of PCBs and PAHs in environment. On the other hand, more attention should be paid to urban sewage treatment process adopting activated-sludge method, where priority organic pollutants might not be removed entirely, even had higher concentrations after treatment. The use of treated sewage should also be controlled carefully.

\subsection{Estimation of aqueous concentrations of target $P C B$ congeners}

Dissolved or biologically available concentration of contaminants is the basis for risk assessment in aquatic ecosystem (Chiou, 1985). Many studies indicate that the uptake of organic compounds by SPMDs is in accordance with polymer permeability and equilibrium partition theory. As chemical partitioning coefficient between membrane and water $\left(K_{\mathrm{MW}}\right)$ and partitioning coefficient between lipid and water $\left(K_{\mathrm{LW}}\right)$ increase with the lipophilic indicator $\left(K_{\mathrm{OW}}\right)$, the SPMD sampling rate for this analytes is expected to rise proportionally. For chemicals having $K_{\mathrm{OW}}$ higher than $10^{3}$ and lower than $10^{5}$, it is observed (Sabaliunas and Sodergren, 1997; Wang et al., 1999). However, when $K_{\mathrm{OW}}$ increases to above $10^{5}$, or molecular size of chemicals approaches and exceeds the size of the average available membrane cavities, a switch to diffusion layer control happens, resistance to mass transfer rises, and $R_{\mathrm{S}}$ declines (Huckins et al., 1997). 


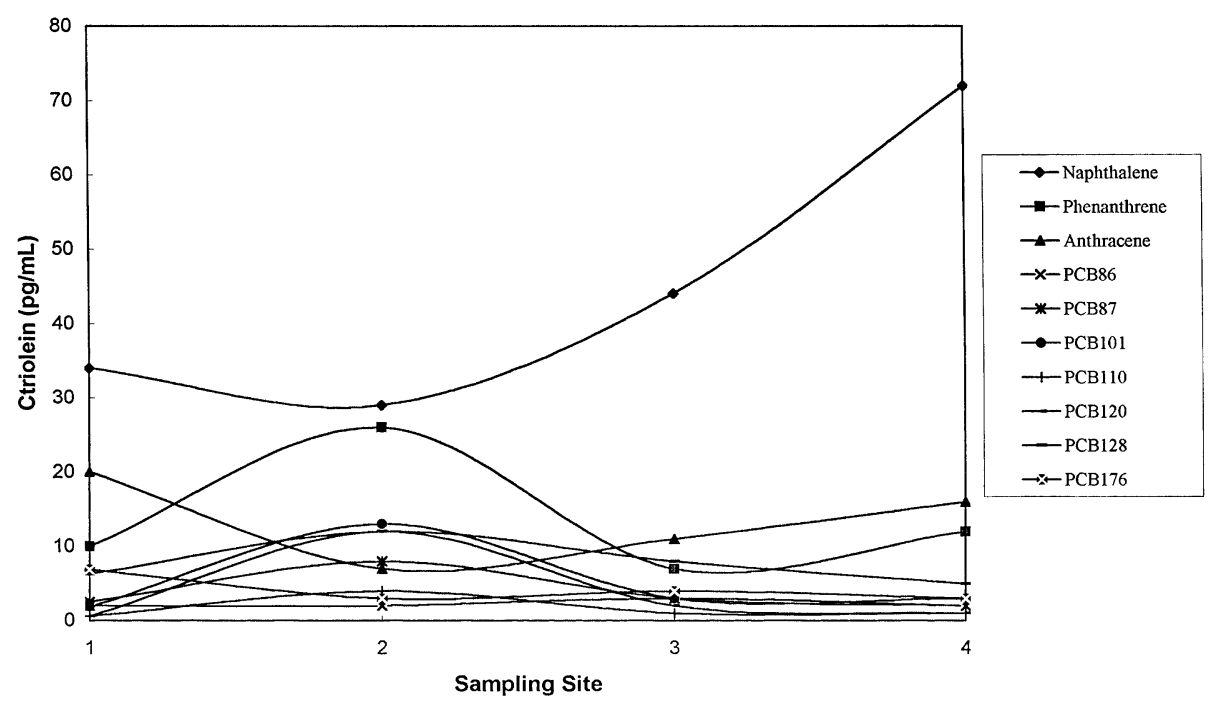

Fig. 2. Variation in concentrations of PAHs and PCB congeners determined in triolein of SPMD along four sampling sites.

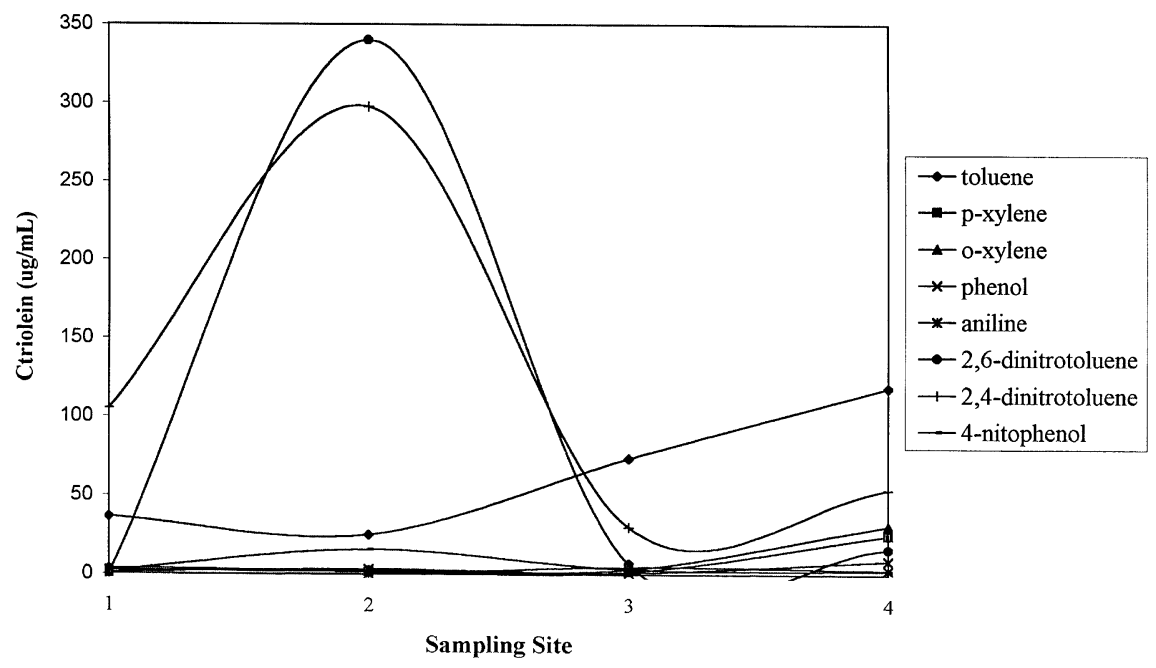

Fig. 3. Variation in concentrations of substituted benzenes determined in triolein of SPMD along four sampling sites.

Huckins et al. (1996b) described the uptake of chemicals by SPMD for which the equilibrium between the SPMD lipid and water is not approached during SPMD exposures (at a constant temperature) in Eq. (1)

$C_{\mathrm{L}}=C_{\mathrm{W}} R_{\mathrm{S}} t / V_{\mathrm{L}}$

where $C_{\mathrm{L}}$ and $C_{\mathrm{W}}$ are the concentrations of a chemical in triolein of SPMDs and water, respectively, $R_{\mathrm{S}}$ the effective daily sampling rate of the SPMD for a particular analyte, in $\mathrm{L} / \mathrm{d}, t$ the sampling time (d), and $V_{\mathrm{L}}$ is the volume of triolein used in the SPMD. The use of Eq. (1) to estimate chemical $C_{\mathrm{W}}$ requires the SPMD to be still in the linear sampling phase or data on the average $R_{\mathrm{S}}$ during the interval of exposure.
In this study, the uptake of PCBs should not approach equilibrium during the 20 day-exposure because of their large $K_{\mathrm{OW}}$ values $\left(>10^{5}\right)$, which was shown in Fig. 4. The uptake of PCB congeners remained in the linear sampling phase. Field mean $R_{\mathrm{S}}$ data of same SPMD configuration determined in our previous work of Yanghe river (Wang et al., 1999) for PCB congeners were used in Eq. (1) together with $C_{\mathrm{L}}$ data to give estimated $C_{\mathrm{W}}$ at each site of sewage treatment process. More field mean $R_{\mathrm{S}}$ data for other target compounds are being determined in our future work in order to test estimation effects further.

Estimated concentrations of PCB congeners from SPMD data were much closed to those determined by dichloromethane extraction for all sampling sites 


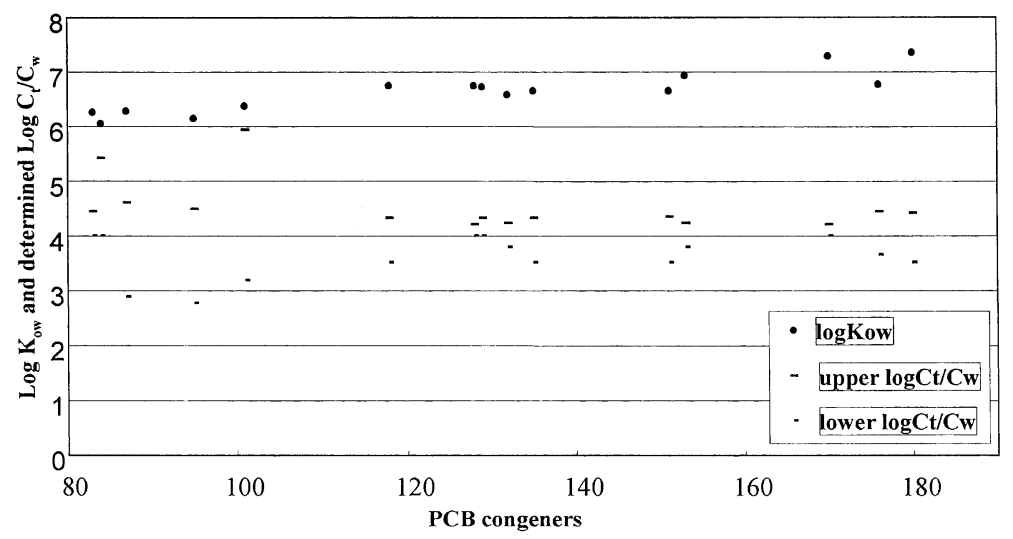

Fig. 4. Comparison of $\log K_{\mathrm{tw}}$ values with the upper and lower limit of $\log C_{\mathrm{t}} / C_{\mathrm{w}}$ for PCB congeners $\left(C_{\mathrm{t}}\right.$ and $C_{\mathrm{w}}$ are concentrations of pollutants in triolein and in sewage, respectively. The upper and lower limits were selected from four sampling sites).

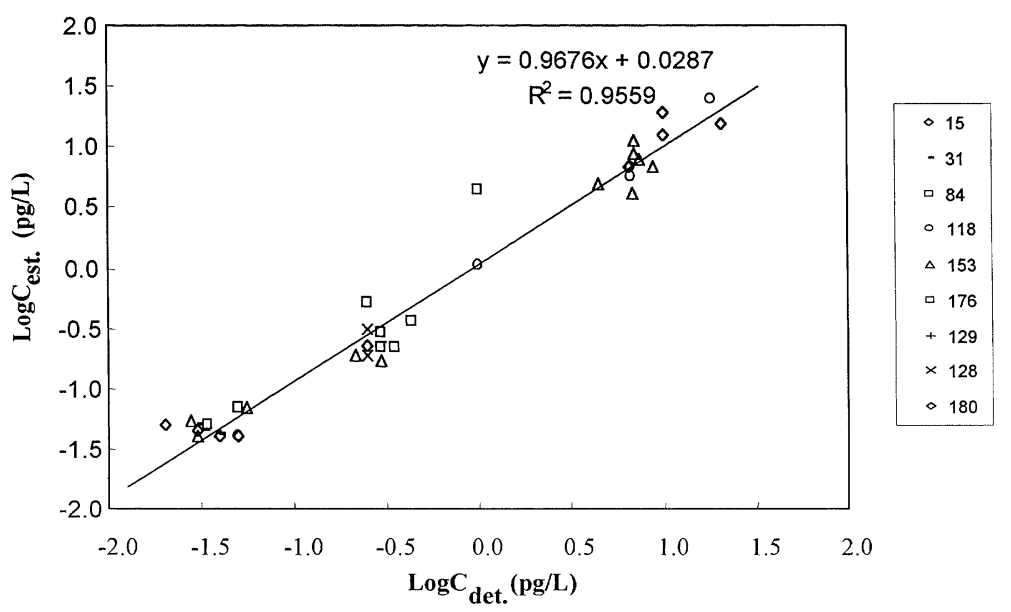

Fig. 5. Plot of logarithm concentrations determined by liquid-liquid extraction versus those estimated by SPMD sampling for PCB congeners.

$\left(R^{2}=0.9559\right)$, as shown in Fig. 5. It should also be noticed that the estimated concentrations of priority pollutants by SPMD sampling reflected biological available forms, while their concentrations in filtered sewage, as extracted by dichloromethane, reflected both dissolved and organic-associated forms. Further attempts are required to optimize this promising methodology, and to increase the accuracy of water concentration estimates for more target contaminants.

\section{Acknowledgements}

This paper was supported by National Nature Science Foundation Project 39770152 and by the Chinese Academy of Sciences. We are grateful for the advice of
Dr. James N. Huckins of Environmental and Contaminants Research Center, USGS, USA.

\section{References}

Bennett, E.R., Metcalfe, T.L., Metcalfe, C.D., 1996. SPMDs for monitoring organic contaminants in the Otonabee river, Ontario. Chemosphere 33 (3), 363-375.

Chiou, C.T., 1985. Partition coefficients of organic compounds in lipid-water systems and correlations with fish bioconcentration factors. Environ. Sci. Technol. 19, 57-62.

Frame, G., 1997. Congener-specific: Comprehensive standard sets combined with improved capillary GC columns and detectors bring complete characterization of PCB mixtures within reach. Anal. Chem. News Features 78, 468A-475A.

Hofelt, C.S., Shea, D., 1997. Accumulation of organochlorine pesticides and PCBs by SPMDs and Mytilus edulis in New Bedford Harbor. Environ. Sci. Technol. 31, 154-159. 
Huckins, J.N., Manuweera, G.K., Petty, J.D., 1993. Liquid containing SPMDs for monitoring organic contaminants in water. Environ. Sci. Technol. 27, 2489-2496.

Huckins, J.N., Petty, J.D., Lebo, J.A., Orazio, C.E., Prest, H.F., 1996a. In: Ostrander, G. (Ed.), Techniques in Aquatic Toxicology. Lewis Publishers, Boca Raton, FL.

Huckins, J.N., Petty, J.D., Meadows, J.C., Echols, K.R., Gale, R.W., 1996b. How chemicals uptake by SPMDs relates to bioconcentration, and basis of comparison. SETAC-1996, 17th Annual meeting, Washington, DC.

Huckins, J.N., Petty, J.D., Prest, H.F., Lebo, J.A., Orazio, C.E., 1997. Important considerations in SPMDs design, application, performance, and data comparability. SETAC1997, 18th Annual meeting, San Francisco, CA.

Sabaliunas, D., Sodergren, A., 1997. Application of SPMDs for preconcentration of organic pollutants and assessment of their effects. Environ. Pollut. 96 (2), 195-205.

Wang, Y., Wang, Z.J., Liu, J.A., Ma, M., Belzile, N., 1999. Monitoring priority pollutants in Yanghe River using dichloromethane extraction and SPMD. Chemosphere 39 (1), 113-131. 